\title{
Recent Developments in the Nasal Immunization against Anthrax
}

\author{
Sandra Jesus ${ }^{1,2}$; Olga Borges ${ }^{1,2}$ * \\ ${ }^{1}$ Center for Neuroscience and Cell Biology, University of Coimbra, Coimbra, Portugal; ${ }^{2}$ Faculty of Pharmacy, University of Coimbra, \\ Pólo das Ciências da Saúde Azinhaga de Santa Comba, Coimbra, Portugal. \\ Email: *olga@ci.uc.pt
}

Received May $30^{\text {th }}, 2011$; revised June $22^{\text {nd }}, 2011$; accepted July $27^{\text {th }}, 2011$.

\begin{abstract}
Bacillus anthracis is the causative agent of anthrax, a bacterial infection with a high mortality rate [1-3]. Although anthrax infection can be cutaneous, gastrointestinal or pulmonary, the pulmonary form is the most deadly [2,3]. Thus, the release of Bacillus anthracis spores that can be inhaled represents a potent bioterrorism threat; the capacity of B. anthracis spores to act as a bioterrorism weapon was demonstrated in 2001, with the intentional infection of 22 persons in the USA [2,4]. Until recently, the available vaccines were developed to confer protection against cutaneous infection; despite this, these vaccines demonstrated experimental efficacy against pulmonary infection in multiple animal models $[1,2]$. Nevertheless, there are many limitations for these vaccines to be considered successful and effective vaccine, including the intensity of the required vaccination schedule, the administration route and the presence of local adverse effects experienced after vaccination $[1,3,5,6]$. To develop more efficient vaccines against pulmonary anthrax, intranasal formulations with adjuvant have been studied. These formulations have advantages because they are easy to administer and because they are expected to induce both systemic and respiratory tract mucosal immune responses. Therefore, the main goal of this review is to compare the different experimental adjuvants used with anthrax antigens and the different approaches regarding the vaccination schedule and consecutive boosters.
\end{abstract}

Keywords: Anthrax, Nasal Immunisation, Vaccine, Adjuvant, Immunopotentiator, Delivery System

\section{Introduction}

Anthrax is a rare bacterial infection that is highly lethal in the inhalational form. The etiological agent is Bacillus anthracis, a gram-positive, rod-shaped, facultative aerobic bacteria capable of generating highly resistant endospores when subjected to adverse environmental conditions [3,5]. Although anthrax infection is primarily observed in herbivorous species, such as goats, sheep and cattle, it can also occur in humans [6,7].

In humans, there are 3 forms of anthrax depending upon the initial site of infection: cutaneous anthrax, gastrointestinal anthrax and pulmonary anthrax $[2,3,8]$. The most common form of anthrax is cutaneous; it is also the least dangerous and normally results from the infection of open skin wounds or abrasions. If untreated, cutaneous anthrax results in death in $20 \%$ of cases. The majority of gastrointestinal anthrax infections result in a fatal systemic disease. However, in some cases, gastrointestinal anthrax infection may lead to meningitis. While cutaneous and gastrointestinal anthrax can arise naturally through direct contact with infected animals, pulmonary anthrax is rarely obtained in this manner [5]. Although there are documented cases of mortality associated with cutaneous and gastrointestinal anthrax, the effectiveness of treatment with antibiotics is high. In contrast, pulmonary anthrax is highly dangerous, with mortality rates of approximately $100 \%[2,3]$. Pulmonary infection is caused by $B$. anthracis spore inhalation and is manifested by the rapid development of non-specific flu-like symptoms that culminate in shock, respiratory arrest and death [5,8-10].

Under adverse environmental conditions, B. anthracis generate spores can remain viable in the environment for years. These small spores, which are approximately 1 to $2 \mu \mathrm{m}$ in size, can be inhaled and deposited within the alveolar spaces of the host respiratory tract. Here, the spores are phagocytosed, predominately by macrophages but also by dendritic cells. In the intracellular environment, the spores germinate into bacilli that subsequently escape from the macrophages, multiply extracellularly in the lymphatic system and spread through the bloodstream where rapid multiplication continues [2,5,11]. Alterna- 
tively, spores phagocytosed by macrophages are transported to peribronchial and mediastinal lymph nodes where they germinate and are later released into the bloodstream $[3,5,9]$. Recently, it was discovered that inhaled spores can establish an initial interaction with the nasal-associated lymphoid tissue (NALT), where they germinated within NALT macrophages [5]. Subsequently, the bacteria spread to the lymph nodes, then to the liver, arriving later in the lungs and bloodstream. Subsequent to bacterial replication, $B$. anthracis exotoxin, which is responsible for the clinical manifestations of the disease in humans, is produced by the vegetative form of the bacteria. The capsule allows the bacteria to resist phagocytosis, ensuring the dissemination of $B$. anthracis throughout the host $[2,6]$.

Based on the mechanism illustrated in Figure 1, protective antigen (PA) is a key antigen for the development of a protective response against anthrax; if PA is inactivated, toxin entry into host cells is compromised [1,5]. It is expected that antibodies against PA block the transport of LF and EF into the cells, protecting the host from anthrax toxins [9]. Confirming this hypothesis, protection against inhalational anthrax challenge can be achieved through passive immunity with the transfer of antibodies against anthrax toxins $[12,13]$. However, an anti-spore immune response in addition to targeting PA activity will most likely trigger events earlier during the establishment of infection, offering additional protection against lethal infection [14], particularly if anti-spore antibodies are present in mucosal tissues that are likely sites of spore entry.

\section{Previous B. anthracis Vaccines}

The available, licensed anthrax vaccines include the an thrax vaccine adsorbed (AVA, trade name BioThrax ${ }^{\circledR}$ ), which is available in the USA, and the anthrax vaccine precipitate (AVP), which is available in the UK; these

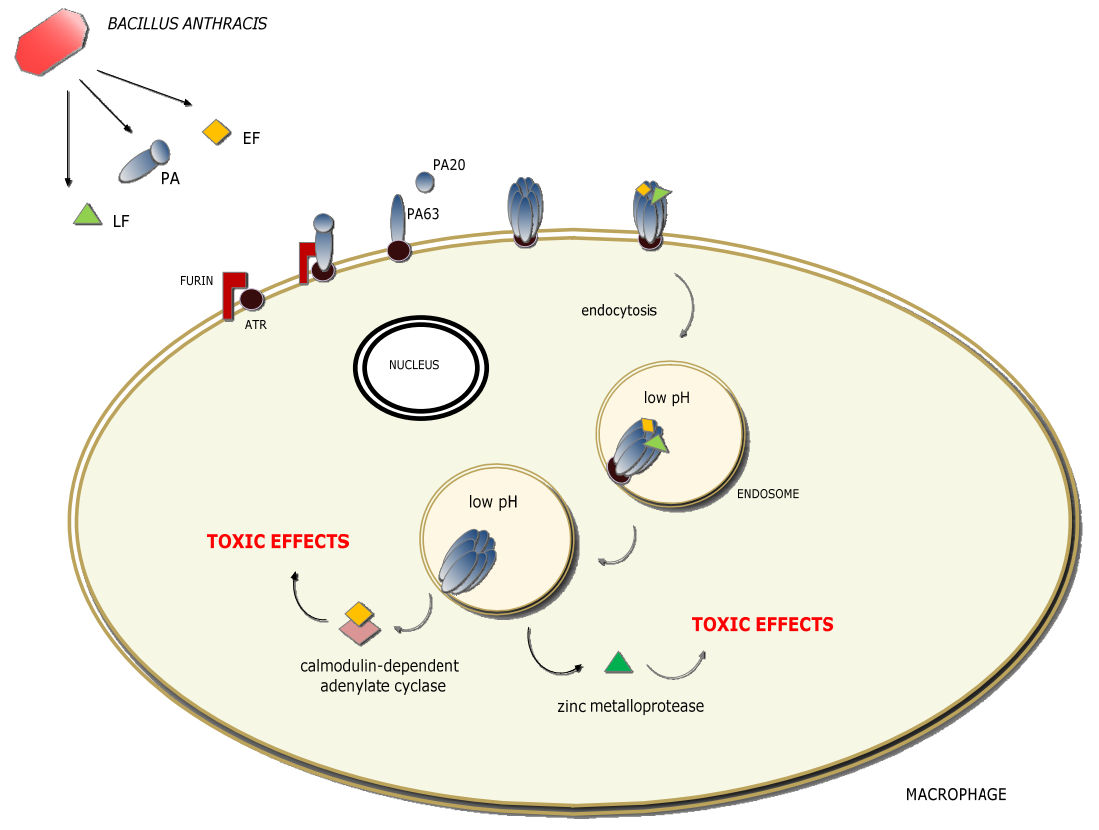

Figure 1. Macrophage infection by Bacillus anthracis (toxaemia stage). Anthrax toxin is comprised of 3 components: oedema factor (EF) and lethal factor (LF), which are A subunits, and protective antigen (PA), which is a B subunit [6,15]. Individually, these three components are not toxic to mammalian cells. However, when in binary combination, they form the following cytotoxic toxins: lethal toxin (PA + LF) and oedema toxin (PA + EF) [6,9,15]. PA binds to cellular receptors, called anthrax toxin receptors (ATR). A furin-like endoprotease cleaves $P A$ and promotes its oligomerisation. The formation of a heptameric PA in the cell membrane functions as an anchor for molecules of lethal factor and/or oedema factor, which bind competitively. The complexes formed on the cell surface are endocytosed and moved into an endosomal compartment with low $\mathrm{pH}$ $[1,12,15,16]$. After acidification, PA forms a pore that translocates the toxins into the cytosol of the host cell where they execute their cytotoxic effects $[5,16]$. Lethal toxin, which is a zinc metalloprotease that inactivates mitogen-activated protein kinase kinase (MAP kinase kinase), causes lysis of susceptible macrophages and loss of alveolar macrophage bactericidal activity, thus facilitating $B$. anthracis survival in the host $[5,6,8]$. Oedema toxin is a calmodulin-dependent adenylate cyclase that catalyses the production of cyclic AMP from host ATP, disturbing the homeostasis of the cell and causing massive oedema. Moreover, it can lead to cell death directly, causing tissue necrosis, multi-organ failure and ultimately host death [5,7]. Thus, these 2 toxins cooperate to inhibit the activation of dendritic cells and $T$ cells, suppressing both innate and adaptive immune response activation [5]. 
vaccines do not inhibit spore germination but neutralise exotoxin, preventing subsequent toxaemia, shock and death $[2,5,7,10]$. AVA contains very small amounts of EF and LF and large quantities of PA adsorbed into aluminium compounds, while AVP contains comparatively lower levels of PA and higher amounts of additional B. anthracis antigens $[2,5,12,17]$.

As these vaccines were originally targeted to protect against cutaneous anthrax, the licensed anthrax vaccines are far from ideal against inhalational anthrax [1,2]. In fact, insufficient data exist regarding their efficacy against inhalation anthrax in humans, although studies in rhesus monkeys indicate that they are protective [18]. Indeed, toxin neutralisation is a main factor for protection against inhalational anthrax. Recently, a group of researchers characterised the levels of antibodies against PA and found that over half of the tested anthrax vaccines elicited responses, whereas serum samples exhibited low levels of toxin neutralisation in vitro [12]. Therefore, current evidence suggests that PA neutralisation alone does not confer optimal protection against anthrax [9]. Many studies suggest that neutralisation of capsule antigens and spore antigens, such as bacillus collagen-like protein of anthracis (BclA), which was recently identified a immunodominant glycoprotein, may provide additional protection to that generated with PA neutralisation $[5,19]$. $B$. anthracis is surrounded by an antiphagocytic polypeptide capsule comprised of poly $\gamma$-D-glutamic acid ( $\gamma$-DPGA or PGA). $\gamma$-DPGA has been identified as a potential target for vaccine development because its disruption can inhibit bacilli growth and subsequent toxin production. However, recent studies have demonstrated that $\gamma$-DPGA is not immunogenic by itself and requires association with carrier proteins or other immunogenic compounds to elicit immune responses $[9,20]$.

Apart from the lack of efficacy already discussed, the available vaccines have several other disadvantages. For instance, the required vaccination schedules are exhausting; for AVA, 6 subcutaneous immunisations spaced throughout the first 18 months and annual booster are required to maintain protection. These vaccines can also cause adverse effects, which have been described indepth within the literature (reviewed in [21]). The less severe symptoms, including local effects such as pain, oedema and erythema, are derived largely from the aluminium compounds used as adjuvants; these side effects are more visible when vaccines are administered by s.c. injection. Aiming to decrease these side effects, in December 2008, the FDA approved a biologics license application (BLA) supplement for use with AVA and reduced its schedule to 5 doses instead of the previous 6 when administered by the intramuscular route [21]. The intentional release of anthrax spores in 2001, which demonstrated the real risk of anthrax spores as a biological weapon, combined with the disadvantages of the existing vaccines led to the realisation for the need to develop an effective vaccine against pulmonary anthrax [6].

Optimal protection against the most dangerous form of anthrax can be improved and ultimately achieved through the induction of specific immunity at spore infection sites, which would lead not only to a systemic immune response but also to the production of antibodies at mucosal surfaces $[9,15]$. Although there are many possible routes of ad-ministration, intranasal administration is most advantageous as it can induce an immune response within the respiratory tract through a simplified and painless procedure [2]. However, the development of an anthrax vaccine for nasal administration is still limited by the need to find suitable and effective adjuvants for this route of administration and type of vaccine [1].

This review will discuss the different possibilities currently under study for the development of a nasally administered anthrax vaccine. Throughout this review, we distinguish between immunopotentiators and antigen delivery systems. This simplistic classification separates adjuvant effects that are derived directly from the potentiation or activation of immune cells, mainly through the Toll-like receptor family, from effects that arise from the transport and delivery of antigens to antigen presenting cells (APC) [22]. The animal models used in the discussed studies vary, making direct comparisons difficult. Additionally, as only non-human primates develop the full range of classic lesions associated with human inhalational anthrax, an effective nasal vaccine in a mouse model may not translate into an efficient vaccine in humans [23,24]. Consistent with this, PA-based vaccines confer better protection in guinea pigs, rabbits and nonhuman primates compared to mice because the $\gamma$ - DPGA capsule is the primary virulence factor in mice [5]. While all animal models represent less than optimal substitutes for human efficacy trials, the rabbit and non-human primate models have been accepted as the best alternatives [7]. Another issue that should be taken into account is the immunisation technique. Although not discussed in-depth in this paper, some protocols included parenteral anaesthesia of the animals with different anaesthetising drugs. The use of anaesthetics with laboratory animals allows for safe animal handling and effective vaccine delivery. Moreover, the use of this technique is known to enhance the immunogenicity of nasally delivered vaccines in mice. In 2010, William M. Gwinn et al. reported that the depth of the anaesthesia influenced vaccine immunogenicity in rabbits due to vaccine retention within the nasal cavity. This observation raises doubts regarding the optimisation of effective nasal vaccines and nasal immunisation strategies using anaesthetised rabbits and their 
applicability to humans [24].

\section{Immune Response to Intranasally Administered Anthrax Vaccine Adsorbed (AVA)}

BioThrax $^{\mathrm{TM}}$ is comprised of aluminium hydroxide-adsorbed compounds from the supernatants of cultures of a toxigenic non-encapsulated B. anthracis strain. The supernatant material predominately contains $\mathrm{PA}$, with undefined quantities of LF, EF and several vegetative cell somatic components. Due to its constitution, BioThrax ${ }^{\mathrm{TM}}$ is expected to prevent toxaemia and apparently septicaemia $[7,15]$. A study published in 2007 aimed to demonstrate that when delivered by the intranasal route, this vaccine would induce both mucosal and systemic immune responses [25]. Additionally, this study also demonstrated that the anti-PA serum antibody response was dose-dependent and that even the lowest dosage tested $\left(7.5 \mu \mathrm{L}\right.$ of BioThrax $\left.{ }^{\mathrm{TM}}\right)$ induced a robust anti-PA $\mathrm{IgG}$, IgG1 and IgG2a response. In regards to antigen-specific mucosal immunity, the dosage of $30 \mu \mathrm{L}$ of BioThrax ${ }^{\mathrm{TM}}$ elicited anti-PA sIgA. Moreover, to confirm the neutralising activity of antibodies against lethal toxins, mice were immunised with different doses of BioThrax ${ }^{\mathrm{TM}}$; dose-dependent survival rates were observed [25]. The authors of the study recognised that more tests were needed in other animal models and that the duration of the protective immunity elicited needed to be studied to conclude if intranasal immunisation with this human anthrax vaccine would provide protection against inhalational anthrax. As B. anthracis culture supernatants contain both antigens and potential immunopotentiators, this could explain the promising results observed. Therefore, the aluminium hydroxide present in this nasal formulation may not have been solely responsible for the observed adjuvant effect. In fact, aluminium compounds have been used exclusively with parenteral vaccines [26] due their low value as mucosal adjuvants.

With the development of a new-generation of vaccines including recombinant subunit and mucosal vaccines that are less immunogenic, the search for more potent vaccine adjuvants has intensified. Recent research has focused on the evaluation of recombinant PA, which would eliminate the need for filtered culture supernatants or whole $B$. anthracis lysates and produce a more consistent vaccine. In fact, Phase I clinical trials of the injectable recombinant vaccine are underway, and the preliminary results for immunogenicity and tolerance have been encouraging [27-29]. Therefore, it is generally accepted that PA, a toxin component from vegetative cells, is central for the design of a human vaccine that targets toxaemia [30]. Phase II clinical trials with injectable rPA adjuvanted with aluminium are underway, and it is predicted that a second generation of anthrax vaccines should be available soon. More recently, great emphasis has been placed on spore antigens, suggesting that neutralisation of these targets contributes to the protection against both toxaemia and septicaemia [31]. Of the more than 40 proteins already identified in the B. anthracis spore coat, immune inhibitor A, GPR-like spore protease and alanine racemase are involved in either germination, spore cytotoxicity, or in the production of glycoprotein BclA from the outermost exosporium layer [32]. All have been selected as potential biological targets to combine with rPA to develop a new vaccine [28-30]. Mucosal administration of rPa combined with other anthrax antigens or with immunopotentiators will constitute the third generation of anthrax vaccines.

\section{Immunopotentiators}

\subsection{Cholera Toxin (CT)}

Cholera toxin (CT), an exotoxin produced by Vibrio cholerae, has been used in animal immunisation experiments as a potent mucosal vaccine adjuvant. The toxicity of CT prevents its use as an adjuvant in clinical trials, but the knowledge of the mechanisms underlying its adjuvant activity may allow for the development of nontoxic mucosal adjuvants for humans use. However, the mechanisms by which CT elicits a mucosal immune response are not completely understood. When administered as an adjuvant, CT increases epithelial permeability, modulates antigen presentation and increases the expression of costimulatory molecules by antigen presenting cells (APC) [6]. It also has been suggested that CT primarily induces Th2-type immune responses characterised by $\mathrm{CD} 4+\mathrm{T}$ cells that produce IL-4, IL-5, IL-6 and IL-10 and by the production of $\operatorname{IgA}$, IgG1 and IgE antibodies [33]. Recently, mice nasally administered with a solution containing recombinant protective antigen (rPA) and cholera toxin (CT) were shown to have generated both systemic and mucosal immune responses. The authors of the study tested 3 concentrations of rPA and 1 concentration of CT, establishing the optimum concentration rPA as $40 \mu \mathrm{g}$. With this formulation, a strong immune response that was predominately $\mathrm{Th} 2$ in nature was observed [15]. In regards to the mucosal immune response, they detected significant levels of anti-rPA secretory $\operatorname{IgA}(\operatorname{sg} \mathrm{A})$ in saliva, nasal washes, vaginal washes and faecal extracts [15]. More recently, it was demonstrated that Th17 cells, a recently identified subset of $\mathrm{CD} 4+\mathrm{T}$ cells that secrete IL-17, mediate the mucosal adjuvant effects of CT [34]. Furthermore, the same study demonstrated that a suspendsion of irradiated anthrax spores (B. anthracis Sterne $\mathrm{pXO}^{1-}, \mathrm{pXO}^{2-}$ ) associated with $1 \mu \mathrm{g}$ CT was able to protect mice against inhalation anthrax. However, de- 
spite the positive results observed using CT as adjuvant, safety issues exist. Intranasal CT administration may lead to the passage of this toxin through the cribriform plate via the olfactory nerve, causing inflammation in the olfactory region of the brain [1]. Thus, investigation of different immunopotentiators for nasal mucosa is urgently required.

\subsection{Polyriboinosinic-Polyribocytidylic Acid}

Polyriboinosinic-polyribocytidylic acid [poly (I:C)] is synthetic double-stranded RNA with immunostimulatory properties $[2,3,35]$ that is recognised by Toll-like recaptor 3 (TLR3). TLR3 is expressed both intracellularly and on the cell surface of fibroblasts and epithelial cells but is localised to the endosomal compartment of myeloid DCs [35]. Therefore, poly (I: C) normally is able to induce type I interferon (IFN), inflammatory cytokine/chemokine production and dendritic cell (DC) maturation via the adaptor protein TICAM-1 (also called TRIF) [35]. When administered to mice nasally at a concentration of $25 \mu \mathrm{g}$ per day for 9 consecutive days or injected intracerebrally at a concentration of $25 \mu \mathrm{g}$, poly (I:C) did not cause any significant side effects. Furthermore, the administration poly (I:C) did not lead to the induction of anti-dsRNA antibodies in the serum of injected animals [3]. Thus far, 3 studies that used poly (I:C) as a nasal vaccine adjuvant in murine anthrax vaccination have been published. The first study involved the immunisation of animals only with PA and poly (I:C). The authors' concluded that poly (I:C) exhibited great potential for mucosal adjuvant; they observed a greater production of anti-PA IgG and IgA and greater anthrax toxin neutralising activity in bronchoalveolar lavage and serum samples when compared to CT [2]. In second study, animals were vaccinated with PA and PGA bound to the protein carrier bovine serum albumin (BSA) and associated to poly (I:C). Anti-PGA antibodies promoted opsonisation of the bacilli by preventing and/or inhibiting bacteraemia, which may constitute a good strategy for the development of a therapeutic vaccine to be used in conjunction with antibiotics following exposure to B. anthracis [3,9]. Finally, the third study, which was published in 2008 [3], showed that nasal immunisation of mice with a prototypic triantigen vaccine candidate comprised of LF, rPA83 and PGABSA associated with poly (I:C) induced strong immune responses against all 3 antigens. Furthermore, the immunised mice survived a challenge with a lethal dose of anthrax. Therefore, neutralisation of LF and PA action are important to promote better protection against the entry of lethal toxins in the host cells. Additionally, anti-PGA antibodies have been demonstrated to exhibit complement-mediated bacteriolytic activity [3]. This study found the optimal dose of poly (I:C) as a nasal vaccine adjuvant to be $10 \mu \mathrm{g}$ per mouse. Also, the authors of the study recognised that further studies will be required to optimise the antigen composition of the vaccine formulation and to evaluate the safety of poly (I:C) as an adjuvant in nasal vaccines in future preclinical and clinical studies.

\subsection{CpG ODN}

CpG oligodeoxynucleotides (ODNs), or synthetic CpGODN, contain un-methylated $\mathrm{CpG}$ motifs and have been used in several studies with the aim of increasing both the humoral and cellular immune responses to vaccines. In particular, for hepatitis B surface antigen, synthetic CpG ODN has been used in preclinical studies as a mucosal adjuvant [36,37] and in early clinical evaluations for its safety, tolerability and immunogenicity when associated with the commercial injectable vaccine [38]. CpG ODN interacts with Toll-like receptor 9 (TLR9), an intracellular receptor expressed by human B cells and plasmacytoid dendritic cells; it triggers the production of proinflammatory cytokines, immunostimulatory chemokines, promote the differentiation of Th1 cells and inducing the functional maturation of antigen presenting cells (reviewed in [39,40]. A study published in 2007 [41] aimed to determine the influence of incorporating $\mathrm{CpG}$ ODN in the AVA vaccine. The effectiveness of this combination was tested after either intraperitoneal (i.p.) or intranasal (i.n.) administration in mice. A previous study from the same authors showed that AVA adjuvanted with CPG ODN administered subcutaneously to macaques signifycantly improved the immune response [42]. Therefore, the hypothesis that the inclusion of CpGODN in intranasal vaccines would increase the immune response was investigated. In fact, the i.n. administration of $20 \mu \mathrm{g}$ of CpG ODN associated AVA vaccine significantly increased levels of anti-PA IgA detected in bronchoalveolar lavage samples and in the gastrointestinal tract, leading to a magnitude of response 3 times higher than what was obtained following i.p. administration. Although, the systemic anti-PA IgG response was also increased due to the presence of $\mathrm{CpG}$ ODN, i.p. immunisation generated antibodies levels 100 times higher compared to i.n. immunisation. When challenged with 20 times the LD50 after 4 weeks of vaccination, the results showed complete protection after injectable vaccination and only $50 \%$ protection following intranasal vaccination [41]. Therefore, this study showed that the intranasal formulation requires further optimisation to obtain better results. It is known that CpG ODN must be co-localised in same APC to generate the most potent therapeutic antigen-specific immune responses. Therefore, delivery vehicles can be utilised to ensure co-delivery of antigens and $\mathrm{CpG}$ ODN to the same APCs, significantly increasing antigen uptake 
by APCs. These strategies can result in antigen-specific immune responses that could be 5- to 500-fold greater compared to administration of the antigen alone [43]. Moreover, for the nasal route of administration, the optimisation of the vaccine formulation is mandatory to obtain improved results.

\subsection{Association of CpG ODN with Chitosan}

Solid nasal formulations are advantageous because they are protected from the antigen degradation that normally occurs in liquid solutions; additionally, they do not require cooling during their shelf life, leading to better transport and storage [44]. During a formulation stability study, it was demonstrated that the stability of a spray freeze-dried (SFD) formulation containing $\mathrm{CpG}$ ODN and rPA that used trehalose as stabiliser was much higher compared to the liquid formulation [44]. Moreover, in particular circumstances, solid formulations may allow for antigen retention on the nasal mucosal surface, increasing the possibility of the antigen uptake by NALT M-cells.

Due to its unique properties, the use of chitosan in nasal vaccine formulations is a research subject of considerable interest. Chitosan is a cationic hydrophilic mucoadhesive polymer that easily forms particles with a good capacity to encapsulate proteins, DNA and antigens. Therefore, beyond its immunostimulatory properties, the ability to encapsulate rPA and CpG ODN in same particle is an attractive quality of chitosan because close physical proximity between antigen and $\mathrm{CpG}$ ODN appears to be advantageous $[44,45]$. Therefore, a chitosanbased powder with $\mathrm{CpG}$ ODN and rPA was tested in rabbits, and the authors reported comparable levels of protection for the i.n. powder vaccine and the injectable vaccine [45].

\subsection{Association of Monophosphoryl Lipid A (MPL) with Chitosan}

Other associations between chitosan and immunopotentiators have been de-scribed in the literature. MPL, a Tolllike receptor 4 (TLR4) agonist, has been extensively evaluated in clinical trials and was proven to be a safe and effective vaccine adjuvant (reviewed in [46]). Thus, MPL was included in a chitosan-based powder formulation and tested with anthrax antigens (described in $[47,48]$ ). In one of the studies, the adjuvant formulation that was evaluated contained the anthrax capsule antigen (10-mer peptide of the poly-D-glutamic acid capsule of B. anthracis) combined with PA. This study showed that the formulations containing chitosan, especially those formulated in powders, generated similar serum levels of anti-PA IgG antibodies compared to the i.m. vaccine. Also, increased anthrax toxin neutralising activity (TNA) was observed only in the powder formulations using chitosan. The immune response induced by capsule antigens is advantageous because the immune response against the PA does not offer an early advantage to eliminate the bacilli responsible for the initial infection. Only the formulations with the capsule protein combined with PA elicited significant levels of anti-capsule IgG antibodies. Therefore, chitosan was shown to have advantages in the generation of anti-PA antibodies and TNA, but not in the generation of anti-capsule antibodies, which depended on the use of the capsule protein in combination with PA. Finally, although all formulations offered full protection 11 weeks after the first immunisation, only the formulation containing MPL, chitosan and PA in conjunction with the capsule antigen resulted in less morbidity among the surviving rabbits [48].

\subsection{Mast Cell (MC) Activators as Adjuvants}

A study published in 2008 by McLachlan et al. examined the role of MC on the induction of antigen-specific humoral immune responses. They used small-molecule MCactivating compounds in conjunction with protein antigens to assess if this combination would result in a potent and protective antigen-specific immune response. The most promising MC-activating compound tested was compound 48/80 (c48/80) [49]. The rationality of this study was based on the knowledge that MCs have key functions in immune response and appear to initiate both the innate immune response and immune cell migration in a coordinated manner, which is believed to be essential in stimulating the adaptive immune response. Mast cells appear to exert these effects by altering the inflammatory environment after pathogen detection by mobilising various immune cells to the site of infection and to the draining lymph nodes [50]. In the McLachlan study, they found that $\mathrm{c} 48 / 80$ induced dendritic cell (DC) and lymphocyte trafficking to the draining lymph nodes (DLNs) indirectly through the activation of NALT mast cells. Furthermore, they demonstrated that $\mathrm{c} 48 / 80$ mediated $\mathrm{T}$ cell-dependent immune responses after nasal administration, and that MCs are required for the induction of humoral immune responses. The intranasal administration of different amounts of c $48 / 80$ plus rPA at days 0,7 and 14 generated an immune response (serum anti-PA IgG and mucosal anti-PA sIgA antibodies, evaluated in vaginal, salivary and faecal extracts) that was comparable to the immune response generated using $\mathrm{CT}$ as an adjuvant. Additionally, no significant differences in IgG isotype titres were observed, and the immune response generated was maintained for at least 6 months. Thus, c48/80 exhibited a potent mucosal adjuvant effect for the induction of both systemic and mucosal immunity by mechanisms independent of TLRs [49]. The adverse effects caused by 
this MC activator were evaluated, and only mild transient irritation was observed; this was especially noted with the highest dose tested $(125 \mu \mathrm{g})$. Taking these results into account and the high prevalence of MCs within the NALT, MC activators can be used in nasal instillation, providing a suitable alternative for future mucosal vaccine adjuvants development [49].

\section{Antigen Delivery Systems}

The adjuvant effect that is achieved through the linking of an antigen to synthetic microparticles has been known for many years and has been previously reviewed in detail $[51,52]$. The delivery of antigens through mucosal membranes remains a major challenge due to unfavourable physiological conditions. To improve mucosal antigen delivery, the use of delivery systems offers numerous advantages, including antigen protection from degradation, increasing antigen concentration in the surrounding mucosal-associated lymphoid tissue, extending their residence time and targeting them to sites of antigen uptake. Within this category of vaccine adjuvants are included adjuvants that mainly function via a "delivery mechanism". However, some delivery systems contain compounds that have immunostimulatory properties. Therefore, due to the appearance of complex vaccine formulations, the classification of vaccine adjuvants is becoming more complicated. The following section describes the main results of studies investigating the use of delivery systems as adjuvants with anthrax antigens.

\subsection{Nanoemulsion}

T_Nanoemulsions (NE) of soybean oil in water $(\mathrm{o} / \mathrm{w})$ exhibit antimicrobial properties are safe and effective and they have proved to have potential for use as vaccine adjuvants. These features have led to the study of NE in a wide variety of vaccines, including vaccines against $\mathrm{Ba}$ cillus anthracis $[1,53]$. It was demonstrated that nanoemulsion-based vaccines were not physically or chemically altered, retained potency following actuation with nasal spray devices [54] and remained stable in terms of appearance and size [1]. Moreover, the same authors found that NE adjuvants induced Th17 (T-helper-17) cytokine responses, which have been recently recognised as an important component in vaccine-induced protective immunity [53]. To test the immune response generated by $\mathrm{rPA}$ mixed with an NE, guinea pig and BALB/c mice were immunised intranasally. The induced immune response was observed at both the systemic and the mucosal level. In the mice, circulating anti-rPA IgG2a and IgG2b antibodies were detected; also, sIgA in bronchoalveolar lavage samples was observed. Immunisation with the NE-rPa formulation also induced high titres of lethal-toxin-neutralising serum antibodies in both mice and guinea pigs. While the immune response triggered by immunisation with NE-rPA was predominately a Th1 response, immunisation with the intramuscular vaccine adjuvanted with aluminium compounds elicited a predominately Th2 immune response [1]. The guinea pig experiments showed that immunisation with concentrations of 10,50 and $100 \mu \mathrm{g}$ rPA mixed with $1 \% \mathrm{NE}$ led to increased IgG levels that lasted for 6 months and give full immunity following intradermal contact with live anthrax spores at a concentration of $1000 \times$ LD50. However, these same immunisations conferred limited protecttion against intranasal contact with live spores. Thus, $70 \%$ of animals survived contact with $10 \times$ LD50 and $40 \%$ survived contact with $100 \times$ LD50; the time to death was delayed 3 - 5 days, with this being the only variable dependent on the concentration of antibodies in serum. According to the authors, this fact may have importance in post-exposure vaccinations that are given in combination with antibiotic or monoclonal antibody therapy [1].

\subsection{Liposome-Protamine-DNA}

Liposome-protamine-DNA (LPD) is an adjuvant that is produced by combining cationic liposomes with an empty plasmid DNA condensed with protamine [55,56]. According to the literature, upon mixing the components spontaneously rearrange to form virus-like particles with condensed DNA inside the liposomes, and rPA should be add to the preparation prior to the spontaneous rearrangement [57]. LPD safety profile was established through a clinical trial conducted in 2000 that is described in Leone et al. [55]. Mice that were i.n. immunised with rPA embedded in LPD exhibited immune responses similar to mice immunised using CT as an adjuvant. With this formulation, high serum levels of anti-rPA IgG and IgM antibodies were detected; further, serum antibodies exhibited strong neutralising activities against lethal toxins. In regards to the mucosal immune response, a significant amount of anti-PA sIgA and a lethal toxin neutralising activity was detected in nasal and lung washes. Although the rPA LPD formulation triggered splenocyte proliferation, it was to a lesser extent compared to the formulation containing CT [58]. Contrary to what would be predicted because there were plasmids in the formulation, immunisation with this adjuvant resulted in a high IgG1:IgG2 ratio, which means higher levels of $\operatorname{IgG} 1$ and a Th2-biased immune response. This is important because rPA has a high affinity for IgG1 antibodies, the same antibody type that also appears following nasal immunisation against rPA associated with CT and intramuscular immunisation using aluminium hydroxide as adjuvant. Moreover, this type of immune response promotes the production of antibodies, which is important for a disease mediated by toxins [58]. 


\subsection{Poly (L-Lactic Acid) Microspheres}

Polymeric particles have also been evaluated as a nasal antigen delivery system. Potential benefits and limitations, mainly related with the physiology of the nasal cavity, have been discussed in several publications (a recent example was published in [59]). Therefore, it was not surprising that this approach also has been tested with anthrax antigens. Poly(L-lactic acid) (PLLA) microparticles were tested to determine if they would protect $\mathrm{rPa}$ from enzyme degradation and allow for sustained antigen release [60]. Intranasal administration of rPa-loaded PLLA particles induced a protective immune response (anti-rPA $\mathrm{IgG}$ ) that was slightly lower compared to i.m. injection of $\mathrm{rPA}$; free rPA was non-immunogenic when administered intranasally. Among the formulations tested intranasally, the best results were obtained with rPA-loaded PLLA microspheres (formulation 1) and with rPA weakly bounded to the particle surface by lyophilisation (formulation 2) [60]. Regardless of the administration route, the predominant antibody subclass was IgG1, indicating a Th2 type immune response [60]. When animals were challenged intranasally with anthrax spores, the mice immunised intranasally with the formulation 1 exhibited a survival rate of $83 \%$, whereas mice immunised with the formulation 2 were $100 \%$ protected. Intramuscular administration alone or in combination with i.n. administration led to a protection rate of $100 \%$. Thus, the authors of the study concluded that with this type of formulation, the optimal immunisation process would be an initial intramuscular vaccination with rPA with aluminium as adjuvant or microencapsulated rPA followed by a second nasal immunisation with microencapsulated rPA [60].

\section{Bacterial Vectors}

\subsection{Spores}

Bacterial spores can be considered as adjuvants for immunisation at mucosal surfaces because they exhibit similar characteristics to microparticulate adjuvants. Also, they exhibit a high heat stability and greater ability to stimulate the innate immune system. Antigens bind to the spore surface that then acts as a carrier, leading to increased immune responses, especially in the case of soluble antigens [61]. Bacillus subtilis can be considered a safe adjuvant as it is being widely used as a probiotic in dietary supplements; additionally, immunisations with dead B. subtilis spores are as effective as immunisations using live spores [61]. The fact that the spores of a Bacillus subtilis strain that expressed anthrax PA protected mice against anthrax toxins led to the proposal to adsorb PA onto spores to immunise mice. Following this princeple, mice were immunised three times intranasally; immunisation resulted in high levels of anti-PA IgG and
sIgA antibodies and neutralising toxin activity. The IgG1: IgG2 ratio was low, indicating a predominantly Th1 type immune response [61].

\subsection{Bacterium}

An immunisation strategy that is currently under development is DNA vaccines. This approach may lead to the longer maintenance of target antigen levels in the vaccinated individual, resulting in a longer-lasting immunological memory [17]. Its management can be performed using bacterial, viral or artificial vectors, such as micro and nanoparticles. The advantages of presenting a bacterium as a vector are mainly the possibility of large-scale production, reduced cost and the possibility of oral administration. However, several disadvantages exist, including the large size of the vector, the possibility of inducing a parallel a specific immune response to vector antigens and the risk of bacterial toxin release if the attenuation of the live vector is not well done. Thus, several bacteria such as Salmonella, Bacillus CalmetteGuerin, Shigella and Listeria monocytogenes have been tested using the most harmless strains $[62,63]$. Salmonella enterica serovar Typhi strains expressing rPA have been utilised in recent studies. The safety of using Salmonella enterica serovar Typhi strains as vectors was well established in a phase II clinical trial [64]; furthermore, they exhibit the desired immunomodulatory activity as a live vaccine against Typhoid and as live vector [65]. In one study, a plasmid with the genes encoding the PA of anthrax fused to a sequence of the alpha-haemolysin gene (hlyA) from E. coli was introduced into the Ty21a strain of Salmonella typhi, an attenuated typhoid vaccine strain licensed for human use.

Cytolytic toxins, most of which are pore-forming proteins, are important virulence factors for many bacterial pathogens. In E. coli, 3 important cytolytic toxins have been identified to date: $\alpha$-haemolysin (HlyA), EHEC haemolysin (EHEC-HlyA, Ehx) and a haemolysin called ClyA, SheA or HlyE. Of these, $\alpha$-haemolysin has been most extensively studied [66].

The encoded and optimised PA had no proteolytic cleavage sites, and its stability increased once it was secreted into the extracellular space of the host. Studies were carried out with female $\mathrm{A} / \mathrm{J}$ mice immunised with three i.p. or i.n. doses. In regards to intranasal administration, two weeks after the last dose, low levels of anti-PA IgG antibodies were observed in serum samples. Anthrax toxin neutralising activity (TNA) was also present at this time point, but its level was also lower compared to i.p. immunisation. Moreover, when PA conjugated to hlyA was expressed, protection levels were $100 \%$ for both i.n. and i.p. immunisations. When PA was 
not fused with this sequence, protection was only about $40 \%$. According to the authors of the study, this sequence allows for PA expression to be targeted extracellularly [67]. Two other studies were performed using the same principle with slight modifications. In one of the studies, immunisation with the live vector Salmonella typhi strain CVD908-htrA that expressed ClyA-PA83 was performed in two different species of monkeys. In both species, the i.n. immunisations with the expression vectors generated modest serum levels of anti-PA IgG antibodies, which increased significantly after intramuscular vaccination with PA adjuvanted with alum; both IgG1 and IgG2 antibodies were detected. In contrast, anthrax toxin neutralising activity was observed at significant levels only 7 days after vaccination with PA. The differences in the responses generated with and without the export protein gene added to PA were not significant. Finally, it was concluded that i.n. priming with $\mathrm{S}$. typhi cells that expressed ClyA-PA83 generated memory cells that resulted in IgG levels and TNA activity that was 100 times higher compared to simple i.m. vaccination [65]. In the second study [68], the Salmonella typhi Ty21a strain was also used to express and export PA via two distinct plasmid-based transport systems: the E. coli HlyA system and the S. typhi ClyA export system. Mice were immunised with 3 intranasal doses and a subsequent intramuscular dose based on rPA. IgG antibody levels increased rapidly after immunisation with the vectors, most significantly in animals where PA was transported to the extracellular space by ClyA. After the intramuscular vaccination, there was a significant increase in IgG levels and increased TNA. This study also noted that despite the high levels of TNA achieved with this procedure, these levels are not as high levels following multiple immunisations with the AVA vaccine (PA adsorbed on aluminium hydroxide). This has a particular importance because the protection against anthrax is now known to be more related to TNA levels than IgG levels in mice, guinea pigs and rabbits [68].

In these studies, expression plasmids with inserted antibiotic resistance genes were used to perform a selection process after their introduction into live vectors. Two problems have been highlighted with this methodology: 1) the constant selective pressure required for maintaining potentially unstable plasmids within live vectors is absent in the host after immunisation, increasing the probability of plasmid loss and reduced immune responses against foreign antigens; 2) the need for the containment of these plasmids within live vectors to prevent the possible spread of antimicrobial resistance genes to other species, such as commensal intestinal microbiota or transient pathogenic bacteria [69].

\section{Conclusions}

Infection caused by Bacillus anthracis spore inhalation is inseparable from a possible bioterrorism attack. In a situation of this kind, the absence of prophylactic measures or a situation of a weak or slow response on a therapeutic level can result in high mortality rates [48]. To contradict these facts, great efforts are being made by the scientific community to develop an effective vaccine against the pulmonary form of anthrax. The different methodologies discussed in this paper commonly support the following benefits of nasal administration: ease of administration, greater possibility of mass immunisation, increased cost effectiveness and the ability to induce a mucosal immune response in addition to the systemic response generated by injectable vaccines $[1,56]$.

Although mucosal vaccination theoretically offers the advantage of inhibiting the initial stages of infection, the most promising approaches need to discover and include other antigens besides PA. One possibility should be the inclusion of antigens from anthrax spores to stop the infection at the site of entry into the host. Although PA exhibits strong immunogenicity, it is not produced until after the spore enters the host and germinates. Thus, it is possible that maximum protection during early stages of the infection cannot be achieved if PA is the sole antigen in a vaccine formulation. In this review, most of the vaccines studied used PA as the sole antigen. Nevertheless, there is literature that suggests that in vitro, anti-PA antibodies stimulate spore up-take and interfere with germination $[7,11,14,25,70]$.

Among formulations already studied for intranasal vaccination, powder vaccine formulations provide additional benefits because they are more stable, easier to handle and do not require temperature control during transport and storage [48].

\section{REFERENCES}

[1] A. U. Bielinska, K. W. Janczak, J. J. Landers, P. Makidon, L. E. Sower, J. W. Peterson, et al., "Mucosal Immunization with a Novel Nanoemulsion-Based Recombinant Anthrax Protective Antigen Vaccine Protects against Bacillus Anthracis Spore Challenge," Infection and Immunity, Vol. 75, No. 8, 2007, pp. 4020-4029. doi:10.1128/IAI.00070-07

[2] B. R. Sloat and Z. Cui, "Nasal Immunization with Anthrax Protective Antigen Protein Adjuvanted with Polyriboinosinic-Polyribocytidylic Acid Induced Strong Mucosal and Systemic Immunities," Pharmaceutical Research, Vol. 23, No. 6, 2006, pp. 1217-1226. doi:10.1007/s11095-006-0206-9

[3] B. R. Sloat, D. S. Shaker, U. M. Le and Z. Cui, "Nasal Immunization with the Mixture of PA63, LF, and a PGA Conjugate Induced Strong Antibody Responses against All Three Antigens," FEMS Immunology and Medical 
Microbiology, Vol. 52, No. 2, 2008, pp. 169-179. doi:10.1111/j.1574-695X.2007.00347.X

[4] D. B. Jernigan, P. L. Raghunathan, B. P. Bell, R. Brechner, E. A. Bresnitz, J. C. Butler, et al., "Investigation of Bioterrorism-Related Anthrax, United States, 2001: Epidemiologic Findings," Emerging Infectious Diseases, Vol. 8, No. 10, 2002, pp. 1019-1028.

[5] Y. P. Gauthier, J. N. Tournier, J. C. Paucod, J. P. Corre, M. Mock, P. L. Goossens, et al., "Efficacy of a Vaccine Based on Protective Antigen and Killed Spores against Experimental Inhalational Anthrax," Infection and Immunity, Vol. 77, No. 3, 2009, pp. 1197-1207. doi:10.1128/IAI.01217-08

[6] H. Sijun and X. Yong, "Helicobacter Pylori Vaccine: Mucosal Adjuvant \& Delivery Systems," Indian Journal of Medical Research, Vol. 130, No. 2, 2009, pp. 115-124.

[7] A. M. Friedlander and S. F. Little, "Advances in the Development of Next-Generation Anthrax Vaccines," Vaccine, Vol. 27, Supplement 4, 2009, pp. D28-D32. doi:10.1016/j.vaccine.2009.08.102

[8] A. K. Pickering, M. Osorio, G. M. Lee, V. K. Grippe, M. Bray and T. J. Merkel, "Cytokine Response to Infection with Bacillus Anthracis Spores," Infection and Immunity, Vol. 72, No. 11, 2004, pp. 6382-6389. doi:10.1128/IAI.72.11.6382-6389.2004

[9] B. R. Sloat and Z. Cui, "Nasal Immunization with a Dual Antigen Anthrax Vaccine Induced Strong Mucosal and Systemic Immune Responses against Toxins and Bacilli," Vaccine, Vol. 24, No. 40-41, 2006, pp. 6405-6413. doi:10.1016/j.vaccine.2006.06.002

[10] Y. Zhang, J. Qiu, Y. Zhou, F. Farhangfar, J. Hester, A. Y. Lin, et al., "Plasmid-Based Vaccination with Candidate Anthrax Vaccine Antigens Induces Durable Type 1 and Type 2 T-Helper Immune Responses," Vaccine, Vol. 26, No. 5, 2008, pp. 614-622. doi:10.1016/j.vaccine.2007.11.072

[11] J. Enkhtuya, K. Kawamoto, Y. Kobayashi, I. Uchida, N. Rana and S. Makino, "Significant Passive Protective Effect against Anthrax by Antibody to Bacillus Anthracis Inactivated Spores That Lack Two Virulence Plasmids," Microbiology, Vol. 152, No. Part 10, 2006, pp. 3103-3110. doi: $10.1099 /$ mic. $0.28788-0$

[12] S. R. Crowe, L. L. Ash, R. J. Engler, J. D. Ballard, J. B. Harley, A. D. Farris, et al., "Select Human Anthrax Protective Antigen Epitope-Specific Antibodies Provide Protection from Lethal Toxin Challenge," Journal of Infectious Diseases, Vol. 202, No. 2, 2010, pp. 251-260. doi:10.1086/653495

[13] S. F. Little, B. E. Ivins, P. F. Fellows and A. M. Friedlander, "Passive Protection by Polyclonal Antibodies against Bacillus Anthracis Infection in Guinea Pigs," Infection and Immunity, Vol. 65, No. 12, 1997, pp. 51715175 .

[14] S. Welkos, S. Little, A. Friedlander, D. Fritz and P. Fellows, "The Role of Antibodies to Bacillus Anthracis and Anthrax Toxin Components in Inhibiting the Early Stages of Infection by Anthrax Spores," Microbiology,
Vol. 147, No. Part 6, 2001, pp. 1677-1685.

[15] P. N. Boyaka, A. Tafaro, R. Fischer, S. H. Leppla, K. Fujihashi and J. R. McGhee, "Effective Mucosal Immunity to Anthrax: Neutralizing Antibodies and Th cell Responses Following Nasal Immunization with Protective Antigen," The Journal of Immunology, Vol. 170, No. 11, 2003, pp. 5636-5643.

[16] J. A. Young and R. J. Collier, “Anthrax Toxin: Receptor Binding, Internalization, Pore Formation, and Translocation," Annual Review of Biochemistry, Vol. 76, 2007, pp. 243-265.

doi:10.1146/annurev.biochem.75.103004.142728

[17] R. J. Cybulski Jr., P. Sanz and A. D. O’Brien, “Anthrax Vaccination Strategies," Molecular Aspects of Medicine, Vol. 30, No. 6, 2009, pp. 490-502.

doi:10.1016/j.mam.2009.08.006

[18] B. E. Ivins, M. L. Pitt, P. F. Fellows, J. W. Farchaus, G. E. Benner, D. M. Waag, et al., "Comparative Efficacy of Experimental Anthrax Vaccine Candidates against Inhalation Anthrax in Rhesus Macaques," Vaccine, Vol. 16, No. 11-12, 1998, pp. 1141-1148. doi:10.1016/S0264-410X(98)80112-6

[19] T. N. Brahmbhatt, B. K. Janes, E. S. Stibitz, S. C. Darnell, P. Sanz, S. B. Rasmussen, et al., "Bacillus Anthracis Exosporium Protein BclA Affects Spore Germination, Interaction with Extracellular Matrix Proteins, and Hydrophobicity," Infection and Immunity, Vol. 75, No. 11, 2007, pp. 5233-5239. doi:10.1128/IAI.00660-07

[20] J. Joyce, J. Cook, D. Chabot, R. Hepler, W. Shoop, Q. Xu, et al., "Immunogenicity and Protective Efficacy of Bacillus Anthracis Poly-Gamma-D-Glutamic Acid Capsule Covalently Coupled to a Protein Carrier Using a Novel Triazine-Based Conjugation Strategy," The Journal of Biological Chemistry, Vol. 281, No. 8, 2006, pp. 48314843. doi:10.1074/jbc.M509432200

[21] J. G. Wright, C. P. Quinn, S. Shadomy and N. Messonnier, "Use of Anthrax Vaccine in the United States: Recommendations of the Advisory Committee on Immunization Practices (ACIP)," MMWR-Recommendations and Reports, Vol. 59, No. RR-6, 2009, pp. 1-30.

[22] V. Schijns and J. Brewer, "Immunopotentiators in Modern Vaccines' (IMV-II) held in Malaga, Spain, May 18-20, 2005," Vaccine, Vol. 24, No. 26, 2006, pp. 5391-5392. doi:10.1016/j.vaccine.2006.03.053

[23] N. A. Twenhafel, "Pathology of Inhalational Anthrax Animal Models," Veterinary Pathology, Vol. 47, No. 5, 2010, pp. 819-830. doi:10.1177/0300985810378112

[24] W. M. Gwinn, S. M. Kirwan, S. H. Wang, K. A. Ashcraft, N. L. Sparks, C. R. Doil, et al., "Effective Induction of Protective Systemic Immunity with Nasally Administered Vaccines Adjuvanted with IL-1," Vaccine, Vol. 28, No. 42, 2010, pp. 6901-6914. doi:10.1016/j.vaccine.2010.08.006

[25] M. Zeng, Q. Xu and M. E. Pichichero, "Protection against Anthrax by Needle-Free Mucosal Immunization with Human Anthrax Vaccine," Vaccine, Vol. 25, No. 18, 2007, pp. 3588-3594. doi:10.1016/j.vaccine.2007.01.075 
[26] T. Ebensen and C. A. Guzman, "Immune Modulators with Defined Molecular Targets: Cornerstone to Optimize Rational Vaccine Design," Human Vaccines, Vol. 4, No. 1, 2008, pp. 13-22. doi: $10.4161 /$ hv.4.1.5560

[27] G. J. Gorse, W. Keitel, H. Keyserling, D. N. Taylor, M. Lock, K. Alves, et al., "Immunogenicity and Tolerance of Ascending Doses of a Recombinant Protective Antigen (rPA102) Anthrax Vaccine: A Randomized, DoubleBlinded, Controlled, Multicenter Trial," Vaccine, Vol. 24, No. 33-34, 2006, pp. 5950-5959. doi:10.1016/j.vaccine.2006.05.044

[28] B. K. Brown, J. Cox, A. Gillis, T. C. VanCott, M. Marovich, M. Milazzo, et al., "Phase I Study of Safety and Immunogenicity of an Escherichia Coli-Derived Recombinant Proective Antigen (rPA) Vaccine to Prevent Anthrax in Adults," PLoS One, Vol. 5, No. 11, p. e13849. doi:10.1371/journal.pone.0013849

[29] J. D. Campbell, K. H. Clement, S. S. Wasserman, S. Donegan, L. Chrisley and K. L. Kotloff, "Safety, Reactogenicity and Immunogenicity of a Recombinant Protective Antigen Anthrax Vaccine Given to Healthy adults," Human Vaccines, Vol. 3, No. 5, 2007, pp. 205-211. doi:10.4161/hv.3.5.4459

[30] D. G. Bouzianas, "Potential Biological Targets of Bacillus Anthracis in Anti-Infective Approaches against the Threat of Bioterrorism," Expert Review of Anti-Infective Therapy, Vol. 5, No. 4, 2007, pp. 665-684. doi: $10.1586 / 14787210.5 .4 .665$

[31] F. Brossier, M. Levy and M. Mock, "Anthrax spores Make an Essential Contribution to Vaccine Efficacy," Infection and Immunity, Vol. 70, No. 2, 2002, pp. 661-664.

[32] J. Kubler-Kielb, E. Vinogradov, H. Hu, S. H. Leppla, J. B. Robbins and R. Schneerson, "Saccharides Cross-Reactive with Bacillus Anthracis Spore Glycoprotein as an Anthrax Vaccine Component," Proceedings of the National Academy of Sciences of the United States of America, Vol. 105, No. 25, 2008, pp. 8709-8712. doi:10.1073/pnas.0803897105

[33] A. M. H. Jan Holmgren, M. Lebens, J.-B. Sun, F. Anjuère and C. Czerkinsky, "Mucosal Adjuvants Based on Cholera Toxin and E. Coli Heat-Labile Enterotoxin," In: D. O'Hagan and V. E. Schijns, Eds., Immunopotentiators in Modern Vaccines, 1st Edition, Elsevier Academic Press, 2006, pp. 235-252.

[34] S. K. Datta, M. Sabet, K. P. Nguyen, P. A. Valdez, J. M. Gonzalez-Navajas, S. Islam, et al., "Mucosal Adjuvant Activity of Cholera Toxin Requires Th17 Cells and Protects against Inhalation Anthrax," Proceedings of the National Academy of Sciences of the United States of America, Vol. 107, No. 23, 2010, pp. 10638-10643. doi:10.1073/pnas.1002348107

[35] M. Matsumoto and T. Seya, "TLR3: Interferon Induction by Double-Stranded RNA Including Poly(I:C)," Advanced Drug Delivery Reviews, Vol. 60, No. 7, 2008, pp. 805-812. doi:10.1016/j.addr.2007.11.005

[36] O. Borges, A. Cordeiro-da-Silva, J. Tavares, N. Santarem, A. de Sousa, G. Borchard, et al., "Immune Response by Nasal Delivery of Hepatitis B Surface Antigen and
Codelivery of a CpG ODN in Alginate Coated Chitosan Nanoparticles," European Journal of Pharmaceutics and Biopharmaceutics, Vol. 69, No. 2, 2008, pp. 405-416. doi:10.1016/i.ejpb.2008.01.019

[37] O. Borges, J. Tavares, A. de Sousa, G. Borchard, H. E. Junginger and A. Cordeiro-da-Silva, "Evaluation of the Immune Response Following a Short Oral Vaccination Schedule with Hepatitis B Antigen Encapsulated into Alginate-Coated Chitosan Nanoparticles," European Journal of Pharmaceutical Sciences, Vol. 32, No. 4-5, 2007, pp. 278-290. doi:10.1016/j.ejps.2007.08.005

[38] C. L. Cooper, H. L. Davis, M. L. Morris, S. M. Efler, M. A. Adhami, A. M. Krieg, et al., "CPG 7909, an Immunostimulatory TLR9 Agonist Oligodeoxynucleotide, as Adjuvant to Engerix-B HBV Vaccine in Healthy Adults: A Double-Blind Phase I/II Study," Journal of Clinical Immunology, Vol. 24, No. 6, 2004, pp. 693-701. doi:10.1007/s10875-004-6244-3

[39] C. Bode, G. Zhao, F. Steinhagen, T. Kinjo and D. M. Klinman, "CpG DNA as a Vaccine Adjuvant," Expert Review of Vaccines, Vol. 10, No. 4, 2011, pp. 499-511. doi:10.1586/erv.10.174

[40] J. Vollmer and A. M. Krieg, "Immunotherapeutic Applications of CpG Oligodeoxynucleotide TLR9 Agonists," Advanced Drug Delivery Reviews, Vol. 61, No. 3, 2009, pp. 195-204. doi:10.1016/j.addr.2008.12.008

[41] D. M. Klinman, D. Currie, G. Lee, V. Grippe and T. Merkel, "Systemic but Not Mucosal Immunity Induced by AVA Prevents Inhalational Anthrax," Microbes and Infection, Vol. 9, No. 12-13, 2007, pp. 1478-1483. doi:10.1016/j.micinf.2007.08.002

[42] D. M. Klinman, H. Xie, S. F. Little, D. Currie and B. E. Ivins, "CpG Oligonucleotides Improve the Protective Immune Response Induced by the Anthrax Vaccination of Rhesus Macaques," Vaccine, Vol. 22, No. 21-22, 2004, pp. 2881-2886. doi:10.1016/j.vaccine.2003.12.020

[43] Y. Krishnamachari and A. K. Salem, "Innovative Strategies for Co-Delivering Antigens and CpG Oligonucleotides," Advanced Drug Delivery Reviews, Vol. 61, No. 3, 2009, pp. 205-217. doi:10.1016/j.addr.2008.12.013

[44] G. Jiang, S. B. Joshi, L. J. Peek, D. T. Brandau, J. Huang, M. S. Ferriter, et al., "Anthrax Vaccine Powder Formulations for Nasal Mucosal Delivery," Journal of Pharmaceutical Sciences, Vol. 95, No. 1, 2006, pp. 80-96. doi:10.1002/jps.20484

[45] J. Huang, J. A. Mikszta, M. S. Ferriter, G. Jiang, N. G. Harvey, B. Dyas, et al., "Intranasal Administration of Dry Powder Anthrax Vaccine Provides Protection against Lethal Aerosol Spore Challenge," Human Vaccines, Vol. 3, No. 3, 2007, pp. 90-93. doi:10.4161/hv.3.3.4011

[46] J. R. Baldridge, P. McGowan, J. T. Evans, C. Cluff, S. Mossman, D. Johnson, et al., "Taking a Toll on Human Disease: Toll-Like Receptor 4 Agonists as Vaccine Adjuvants and Monotherapeutic Agents," Expert Opinion on Biological Therapy, Vol. 4, No. 7, 2004, pp. 11291138. doi:10.1517/14712598.4.7.1129

[47] S. D. Klas, C. R. Petrie, S. J. Warwood, M. S. Williams, 
C. L. Olds, J. P. Stenz, et al., "A Single Immunization with a Dry Powder Anthrax Vaccine Protects Rabbits against Lethal Aerosol Challenge," Vaccine, Vol. 26, No. 43, 2008, pp. 5494-5502.

doi:10.1016/i.vaccine.2008.07.062

[48] S. Wimer-Mackin, M. Hinchcliffe, C. R. Petrie, S. J. Warwood, W. T. Tino, M. S. Williams, et al., "An Intranasal Vaccine Targeting Both the Bacillus Anthracis Toxin and Bacterium Provides Protection against Aerosol Spore Challenge in Rabbits," Vaccine, Vol. 24, No. 18, 2006, pp. 3953-3963. doi:10.1016/j.vaccine.2006.02.024

[49] J. B. McLachlan, C. P. Shelburne, J. P. Hart, S. V. Pizzo, R. Goyal, R. Brooking-Dixon, et al., "Mast Cell Activators: A New Class of Highly Effective Vaccine Adjuvants," Nature Medicine, Vol. 14, No. 5, 2008, pp. 536-541. doi:10.1038/nm1757

[50] S. N. Abraham and A. L. St John, "Mast Cell-Orchestrated Immunity to Pathogens," Nature Reviews Immunology, Vol. 10, No. 6, 2010, pp. 440-452. doi:10.1038/nri2782

[51] B. C. Baudner and D. T. O'Hagan, "Bioadhesive Delivery Systems for Mucosal Vaccine Delivery," Journal of Drug Targeting, Vol. 18, No. 10, pp. 752-770. doi:10.3109/1061186X.2010.529143

[52] N. Mishra, A. K. Goyal, S. Tiwari, R. Paliwal, S. R. Paliwal, B. Vaidya, et al., "Recent Advances in Mucosal Delivery of Vaccines: Role of Mucoadhesive/Biodegradable Polymeric Carriers," Expert Opinion on Therapeutic Patents, Vol. 20, No. 5, 2010, pp. 661-679.

[53] A. U. Bielinska, M. Gerber, L. P. Blanco, P. E. Makidon, K. W. Janczak, M. Beer, et al., "Induction of Th17 Cellular Immunity with a Novel Nanoemulsion Adjuvant," Critical Reviews in Immunology, Vol. 30, No. 2, pp.189-199.

[54] P. E. Makidon, S. S. Nigavekar, A. U. Bielinska, N. Mank, A. M. Shetty, J. Suman, et al., "Characterization of Stability and Nasal Delivery Systems for Immunization with Nanoemulsion-Based Vaccines," Journal of Aerosol Medicine and Pulmonary Drug Delivery, Vol. 23, No. 2, 2010, pp. 77-89.

[55] P. Leone, C. G. Janson, L. Bilaniuk, Z. Wang, F. Sorgi, L. Huang, et al., "Aspartoacylase Gene Transfer to the Mammalian Central Nervous System with Therapeutic Implications for Canavan Disease," Annals of Neurology, Vol. 48, No. 1, 2000, pp. 27-38. doi:10.1002/1531-8249(200007)48:1<27::AID-ANA6 $>3$. $\underline{0 . \mathrm{CO} ; 2-6}$

[56] B. R. Sloat and Z. Cui, "Strong Mucosal and Systemic Immunities Induced by Nasal Immunization with Anthrax Protective Antigen Protein Incorporated in LiposomeProtamine-DNA Particles," Pharmaceutical Research, Vol. 23, No. 2, 2006, pp. 262-269. doi:10.1007/s11095-005-9078-7

[57] Z. Cui and L. Huang, "Liposome-Polycation-DNA (LPD) Particle as a Carrier and Adjuvant for Protein-Based Vaccines: Therapeutic Effect against Cervical Cancer," Cancer Immunology, Immunotherapy, Vol. 54, No. 12, 2005, pp. 1180-1190. doi:10.1007/s00262-005-0685-2
[58] B. R. Sloat, M. A. Sandoval, A. M. Hau, Y. He and Z. Cui, "Strong Antibody Responses Induced by Protein Antigens Conjugated onto the Surface of Lecithin-Based Nanoparticles," Journal of Controlled Release, Vol. 141, No. 1, 2010, pp. 93-100. doi:10.1016/i.jconrel.2009.08.023

[59] J. Ali, M. Ali, S. Baboota, J. K. Sahani, C. Ramassamy, L. Dao, et al., "Potential of Nanoparticulate Drug Delivery Systems by Intranasal Administration," Current Pharmaceutical Design, Vol. 16, No. 14, pp. 1644-1653. doi: $10.2174 / 138161210791164108$

[60] H. C. Flick-Smith, J. E. Eyles, R. Hebdon, E. L. Waters, R. J. Beedham, T. J. Stagg, et al., "Mucosal or Parenteral Administration of Microsphere-Associated Bacillus Anthracis Protective Antigen Protects against Anthrax Infection in Mice," Infection and Immunity, Vol. 70, No. 4, 2002, pp. 2022-2028. doi:10.1128/IAI.70.4.2022-2028.2002

[61] J. M. Huang, H. A. Hong, H. Van Tong, T. H. Hoang, A. Brisson and S. M. Cutting, "Mucosal Delivery of Antigens Using Adsorption to Bacterial Spores," Vaccine, Vol. 28, No. 4, 2010, pp. 1021-1030. doi:10.1016/j.vaccine.2009.10.127

[62] T. D. Nandedkar, "Nanovaccines: Recent Developments in Vaccination," Journal of Biosciences, Vol. 34, No. 6, 2009, pp. 995-1003. doi:10.1007/s12038-009-0114-3

[63] S. S. Rao, D. Styles, W. Kong, C. Andrews, J. P. Gorres and G. J. Nabel, "A Gene-Based Avian Influenza Vaccine in Poultry," Poultry Science, Vol. 88, No. 4, 2009, pp. 860-866. doi: $10.3382 /$ ps.2008-00360

[64] C. O. Tacket, M. B. Sztein, S. S. Wasserman, G. Losonsky, K. L. Kotloff, T. L. Wyant, et al., "Phase 2 Clinical Trial of Attenuated Salmonella Enterica Serovar Typhi Oral Live Vector Vaccine CVD 908-htrA in U.S. Volunteers," Infection and Immunity, Vol. 68, No. 3, 2000, pp. 1196-1201. doi:10.1128/IAI.68.3.1196-1201.2000

[65] J. E. Galen, M. Chinchilla, M. F. Pasetti, J. Y. Wang, L. Zhao, I. Arciniega-Martinez, et al., "Mucosal Immunization with Attenuated Salmonella Enterica Serovar Typhi Expressing Protective Antigen of Anthrax Toxin (PA83) Primes Monkeys for Accelerated Serum Antibody Responses to Parenteral PA83 vaccine," Journal of Infectious Diseases, Vol. 199, No. 3, 2009, pp. 326-335. doi:10.1086/596066

[66] A. Ludwig, S. Bauer, R. Benz, B. Bergmann and W. Goebel, "Analysis of the SlyA-Controlled Expression, Subcellular Localization and Pore-Forming Activity of a 34 kDa Haemolysin (ClyA) from Escherichia coli K-12," Molecular Microbiology, Vol. 31, No. 2, 1999, pp. 557567. doi:10.1046/j.1365-2958.1999.01196.x

[67] M. Osorio, Y. Wu, S. Singh, T. J. Merkel, S. Bhattacharyya, M. S. Blake, et al., "Anthrax Protective Antigen Delivered by Salmonella Enterica Serovar Typhi Ty21a Protects Mice from a Lethal Anthrax Spore Challenge," Infection and Immunity, Vol. 77, No. 4, 2009, pp. 1475-1482. doi:10.1128/IAI.00828-08

[68] L. W. Baillie, A. L. Rodriguez, S. Moore, H. S. Atkins, C. 
Feng, J. P. Nataro, et al., "Towards a Human Oral Vaccine for Anthrax: The Utility of a Salmonella Typhi Ty21a-Based Prime-Boost Immunization Strategy," Vaccine, Vol. 26, No. 48, 2008, pp. 6083-6091.

doi:10.1016/i.vaccine.2008.09.010

[69] J. E. Galen, J. Y. Wang, M. Chinchilla, C. Vindurampulle, J. E. Vogel, H. Levy, et al., "A New Generation of Stable, Nonantibiotic, Low-Copy-Number Plasmids Improves Immune Responses to Foreign Antigens in Salmonella Enterica Serovar Typhi Live Vectors," Infection and
Immunity, Vol. 78, No. 1, 2010, pp. 337-347. doi:10.1128/IAI.00916-09

[70] C. K. Cote, C. A. Rossi, A. S. Kang, P. R. Morrow, J. S. Lee and S. L. Welkos, "The Detection of Protective Antigen (PA) Associated with Spores of Bacillus Anthracis and the Effects of Anti-PA Antibodies on Spore Germination and Macrophage Interactions," Microbial Pathogenesis, Vol. 38, No. 5-6, 2005, pp. 209-225. doi:10.1016/j.micpath.2005.02.001 